\title{
DESARROLLO COMUNITARIO \\ Y CALIDAD DE VIDA ${ }^{1}$
}

Ana Teresa López de Llergo

Luz María Cruz de Galindo

\section{RESUMEN}

Este escrito relaciona el proceso de desarrollo de una comunidad con las distintas categorías que expresan la calidad de vida. Considera al desarrollo comunitario como un proceso que beneficia a cada miembro de la comunidad y la comunidad en su conjunto. Esto es posible siempre y cuando se respete su característica fundamental: la participación activa de los destinatarios que se convierten, así, en actores de su propia mejora. Pero tal desarrollo requiere de un marco conceptual que proporcionará la dirección del proceso y sus metas a corto, mediano y largo plazo. La finalidad última del proceso es el logro de una calidad de vida que no se refiera únicamente a aspectos económicos sino que considere la integralidad del ser humano.

\section{DEFINICIÓN DE CONCEPTOS: COMUNIDAD, DESARROLLO, DESARROLLO COMUNITARIO, ANIMACIÓN SOCIOCULTURAL Y CALIDAD DE VIDA}

\subsection{COMUNIDAD}

La palabra comunidad denota la cualidad de común, o bien la posesión compartida de algo (propósito, espacio territorial, etcétera). Hay variadísimos tipos de comunidades según se clasifiquen por tamaño, ubicación, manera de satisfacer sus

${ }^{1}$ Ponencia presentada en la tercera edición de la Universidad Internacional Ciencia y Vida, Conciencia Cívica y Vida Digna para Todos, México, D.F. del 23 al 27 de agosto de 2000 . 
requerimientos, comunicaciones... Pero todas ellas -para serlo- han de poseer ciertas características²:

- Disponer de un área geográfica definida.

- Que entre sus miembros existan lazos de parentesco.

- Compartir los mismos intereses.

- Tener antecedentes comunes y participar de una misma tradición histórica.

- Que cada uno considere los problemas de la comunidad como propios: sentido de pertenencia al grupo.

- Relaciones cara a cara entre los miembros de la comunidad.

- Ser tributarios de un cuerpo de instituciones y servicios.

La interacción se da dentro de las comunidades y por las interrelaciones de unas con otras. Esto origina, en cada caso, una estructura sociocultural y un modo concreto de satisfacer todo tipo de necesidades.

Las unidades sociales se clasifican en grupos primarios o básicos —familia, amigos, compañeros- y secundarios —barrio, escuela, parroquia, club-. Ambos tienen como telón de fondo la sociedad global. La persona vive dentro de los grupos primarios, y completa su socialización participando en los secundarios. La comunidad es un grupo secundario. Frente a la sociedad fría e impersonal, la comunidad está formada por instituciones e individuos con los cuales nos relacionamos - directa y afectivamente- y compartimos valores dentro de un espacio vital común en el que participamos de ciertas vivencias colectivas.

Ahí se aglutinan intereses y emociones que conforman el sentimiento comunitario y favorecen la cohesión social. La comunidad es - para las personas y los grupos- una importante unidad de vida y de relación, que complementa nuestra identidad personal, nos da identidad social y nos proporciona servicios y, con ello, seguridad ${ }^{3}$.

${ }^{2}$ ESCALANTE F. Rosendo et.al., Investigación, Organización y Desarrollo de la Comunidad, p.34.

3 Cfr. QUINTANA CABANAS, José María. Los Ámbitos Profesionales de la Animación, p.69 y ss. 
Comunidad es una agrupación o conjunto de personas que habitan un espacio geográfico delimitado y delimitable, cuyos miembros tienen conciencia de pertenencia o de identificación con algún símbolo local y que interaccionan entre sí más intensamente que en otro contexto, operando en redes de comunicación, intereses y apoyo mutuo, con el propósito de alcanzar determinados objetivos, satisfacer necesidades, resolver problemas o desempeñar funciones sociales relevantes a nivel local ${ }^{4}$.

Una de las comunidades más interesantes es el municipio, pues constituye un conjunto organizado de servicios y tiene representatividad y libertad de acción. Su estructura social no es horizontalmente plana, hay una dimensión vertical - la estratificación social- que diferencia a sus miembros en términos de superioridad, inferioridad e igualdad 5 . Esta valoración coloca a las personas en distintas capas o estratos y es una de las oportunidades para ejercer el liderazgo.

\subsection{DESARROLLO}

En sentido lato, desarrollo significa crecimiento, progreso, incremento con incidencia en lo personal y comunitario. Se trata de un desarrollo armónico si un nivel complementa al otro, y es muy importante vincular ambos. La historia ha demostrado que esta posición no es fácil —especialmente en la práctica一, pues se tiende a enaltecer los derechos individuales o a negarlos por completo para fortalecer al grupo. En el primer caso se propicia el individualismo; en el segundo, el colectivismo.

Cuando el desarrollo se refiere a los hechos sociales ${ }^{6}$, hablamos de un movimiento que alude no sólo al aspecto económico, sino a todo lo relacionado con los ámbitos de la cultura, materiales, afectivos y espirituales. En el plano material

\footnotetext{
${ }^{4}$ ANDER-EGG, Ezequiel. Metodología y Práctica del Desarrollo Comunitario. ¿Qué es el Desarrollo de la Comunidad?, Vol. 1, p.33, 34.

${ }_{5}^{5}$ Cfr. ESCALANTE-MIÑANO. Op. cit., p.66.

${ }^{6}$ Cfr. Idem, p. 69, 70.
} 
significa soporte técnico, civilización y desenvolvimiento económico, para elevar el nivel de vida de los distintos sectores que la componen. En lo espiritual implica movilidad cultural, desenvolvimiento de la organización social y ampliación de los horizontes científicos, filosóficos y artísticos.

\subsection{DESARROLLO COMUNITARIO}

En 1956, un grupo de expertos de las Naciones Unidas, en la considerada Carta Magna del Desarrollo de la Comunidad, nos señala que tal desarrollo se relaciona con: «aquellos procesos en cuya virtud los esfuerzos de una población se suman a los de su gobierno para mejorar las condiciones económicas, sociales y culturales de las comunidades, integrar éstas a la vida del país y permitirles contribuir plenamente al progreso nacional ${ }^{7}$.

Carolina Ware (1954) habla de "un proceso para suscitar grupos funcionales de ciudadanos capaces de ser agentes activos y responsables de su propio progreso, usando para ello como medios, la investigación en común de los problemas locales, el planeamiento y la ejecución por sí mismos de las soluciones que antes convinieron, y la coordinación voluntaria con los demás grupos y con las autoridades oficiales, de modo que se obtenga el bienestar total de la comunidad ${ }^{8}$.

Sobre la base de las definiciones anteriores y los cambios que a lo largo del tiempo se han dado en esta forma de intervención social, podemos afirmar que el desarrollo comunitario es un proceso que suma los esfuerzos de los pobladores de una región, de los grupos intermedios -escuelas, empresas, clubs, etcétera-y del gobierno, para mejorar las condiciones económicas, sociales y culturales de las comunidades, mediante el diagnóstico, plan, ejecución y evaluación de programas y proyectos para este fin.

${ }^{7}$ Cfr. ANDER-EGG, E., Reflexiones en torno a los Métodos del Trabajo Social, p.47.

${ }^{8}$ ANDER-EGG, E. Idem, p. 48. 
En los años noventa ${ }^{9}$ ya no se habla del papel del desarrollo de la comunidad en la planificación y ejecución del desarrollo nacional, pues cuando los programas se orientan a poblaciones de menos ingresos o marginadas, la preocupación central es promover estrategias de supervivencia, más que hacer una contribución al progreso nacional. Actualmente las pretensiones son más modestas y realistas: ver en qué forma, cómo y con qué proyectos específicos articular esta metodología en la acción local. El desarrollo comunitario se visualiza hoy como una manera de sensibilizar y motivar a las personas para que participen en la solución de sus problemas, y como forma de devolver parcelas de la gestión pública al ámbito de la sociedad civil.

Es por tanto un movimiento mundial ${ }^{10}$ con características especiales para cada país. Como proceso tiende al mejoramiento colectivo de la comunidad, a través de la promoción individual y solidaria de sus integrantes para un fin común; como técnica, se sustenta en las ciencias sociales: Economía, Demografía, Sociología, Antropología, Historia, Psicología y, fundamentalmente, en el conocimiento del proceso educativo. Su acción es multifacética y se expresa en programas cuya finalidad es lograr mejoras concretas. Más que una acción sobre la comunidad, es una acción de la comunidad. Se trata de esfuerzos y acciones de base organizadas con la iniciativa y dirección de los mismos involucrados, aunque para su despegue se necesiten agentes externos, y durante su ejercicio, asistencia técnica.

Cuando esta actividad se sustenta en el proceso educativo, provoca la toma de conciencia del ineludible papel que se ha de desempeñar, apoyándose en las potencialidades latentes de individuos, grupos y comunidades. Así, la educación puede ayudar a satisfacer necesidades cotidianas de la comunidad y favorecer su desarrollo, a través de un aumento cualitativo

${ }^{9}$ Cfr. Idem, Metodología y Práctica del Desarrollo de la Comunidad, p.118, 119.

${ }^{10}$ Cfr. ESCALANTE, F. Op. cit., p.78, 79. 
y cuantitativo de conocimientos teórico-prácticos que permitan a los integrantes del grupo social mejorar sus condiciones de vida. La finalidad será lograr modos de participación que culminen en una organización autogestionaria donde, en el ámbito local y de organizaciones, las personas sean responsables de su proceso de mejora.

La parte operativa —-métodos y técnicas de actuación- ha de contar con recursos pedagógicos y didácticos para provocar y estimular el cambio individual y sociocultural. La idea es fortalecer el sentimiento comunitario y capacitar a cada cual para el esfuerzo cooperativo, con el fin de superar limitaciones - personales y sociales- y actualizar posibilidades. Por eso, la actitud con que se llevan a cabo los proyectos y la forma de emprender el trabajo, son más importantes que el contenido material de los mismos.

Existen dos elementos esenciales en esta práctica social:

1. La participación activa y solidaria de la población para mejorar su calidad de vida.

2. La intervención de instituciones gubernamentales, no gubernamentales y voluntarias, para suministrar ayudas de todo tipo que aumenten la eficacia y capitalicen el esfuerzo y el trabajo de los integrantes de la comunidad.

Sin embargo, es importante evitar dos suposiciones erróneas ${ }^{11}$ :

- Que las comunidades son realidades homogéneas con intereses compartidos por todos sus miembros.

- Que todas las personas quieren mejorar su comunidad y porporcionan los medios para ello.

En un mismo ámbito territorial, pese al sentido de identidad y pertenencia, hay diversidad de actores -individuos, grupos, organizaciones y redes- que se expresan en una gran variedad de modos de actuar y de intereses contrapuestos. En el trasfondo de todo ello subyacen las clases sociales.

${ }^{11}$ Cfr. ANDER-EGG, E.Metodología y Práctica del Desarrollo de la Comunidad, p.99. 
"Consenso y conflicto, cooperación y disociación se dan indefectiblemente a lo largo de los procesos de acción comunitaria " ${ }^{12}$.

La participación será una realidad si se neutralizan las fuerzas negativas, prevalece lo común y hay grupos —institucionales, naturales, formales, informales, etcétera- a los que todos tengan acceso, pues el trabajo comunitario es trabajo con grupos que están unidos por objetivos comunes ${ }^{13}$. Cada grupo ha de ser una unidad de trabajo con tareas concretas, pero abierto a la acción comunitaria. Para ello hace falta un plan - apoyado en un marco teórico referencia ${ }^{14}$ y en la investigación diagnóstica - que coordine y programe las distintas actividades con el fin de lograr las metas propuestas. Así será posible vertebrar a todos los grupos, con la ayuda de los líderes sociales, y producir un movimiento coordinado y total. Para elaborar el plan —y los programas correspondientes - el punto de partida es conocer lo mejor posible a la comunidad en la que se pretende intervenir ${ }^{15}$. Se trata de un conocimiento que lleva a la acción y se retroalimenta de ella. Por eso es necesario vincular el marco teórico, la metodología y la actividad práctica. Vale la pena insistir en algunos puntos básicos ${ }^{16}$ para que este proceso deje huella. Aunque se propusieron a mediados del siglo XX, creemos que no han perdido actualidad:

- Las comunidades pueden desarrollar la capacidad para resolver sus problemas.

- Las personas quieren y pueden cambiar.

- La gente debe participar en los cambios que se realizan en sus comunidades.

\footnotetext{
${ }^{12}$ ANDER-EGG, E. Idem, p.100, 101.

${ }^{13}$ Cfr. KISNERMAN, Natalio. La Comunidad, p.75.

${ }_{14}$ El marco teórico delimita conceptos y señala los ámbitos de la praxis metodológica.

${ }^{15}$ Es muy recomendable que los primeros proyectos busquen satisfacer los deseos expresados de la población. Hay que distinguir también entre las llamadas necesidades sentidas y las reales.

${ }^{16}$ Cfr. MURRAY G. Ross. Community Organization. Theory, Principles and Practices, en ANDER-EGG, E. Metodología y Práctica del Desarrollo Comunitario, p.57.
} 
- El autodesarrollo de una comunidad tiene un significado diferente al de los cambios impuestos.

- Para un buen trabajo comunitario hay que hacer un abordaje bolístico de los problemas; los abordajes fragmentarios son poco eficaces.

- La democracia requiere participación y acciones de cooperación en los asuntos de la comunidad; las personas deben prepararse para hacer esto posible.

- Las comunidades y los individuos necesitan ayuda externa para afrontar la solución de sus problemas y necesidades. Lo importante es que la responsabilidad personal se convierta en responsabilidad social, sin perder aquélla, y se exprese tangiblemente en la calidad de vida. Así, la promoción del desarrollo se llevará a cabo en un clima de respeto a los valores y a las tradiciones del contexto cultural de cada nación, y sin violentar su legítima soberanía.

\subsection{ANIMACIÓN}

El término — sinónimo de vitalización — viene de la palabra anima que significa vida. La realidad de la animación es esencial en el ser humano y ha de proyectarse en lo social. Los ámbitos de la animación se clasifican según ${ }^{17}$ :

1. Los destinatarios o grupos sociales a los que se dirige: niños, jóvenes, adultos, campesinos, etcétera.

2. Los territorios o lugares donde se pretende introducir: barrios, municipios, asociaciones voluntarias, escuelas, centros cívicos.

3. Los hábitats humanos o territorios diferenciales: medio rural, urbano, lugares de vacaciones...

4. Las actividades: artísticas, culturales, sociales, políticas.

5. Los objetivos que la actividad animadora pretende fomentar: promoción cultural, desarrollo social y económico, cultivo de las tradiciones, cambio social, expresión personal, creatividad artística.

${ }^{17}$ Cfr. QUINTANA CABANAS, José María. Op. cit, p.11. 
Por ejemplo, puede hablarse de una animación dirigida a todas las personas -independientemente de su sexo, edad, formación académica - ubicadas en un municipio del medio rural, para promover actividades que favorezcan el desarrollo socioeconómico y cultural de la comunidad en cuestión.

No se trata de que los proyectos de animación sean espectaculares. Lo importante es mejorar la vida cotidiana a partir de lo que es posible. En todos los casos, el animador tiene más movilidad para la acción comunitaria cuando logra armonizar y coordinar los intereses particulares y colectivos, la identidad individual y los procesos sociales, el altruismo y el egoísmo que coexisten en una misma persona o grupo. Para ello, ha de buscar la colaboración de ciudadanos sensibles, conscientes, responsables y dispuestos a dedicar parte de su tiempo a prestar un servicio comunitario ${ }^{18}$.

\subsection{ANIMACIÓN SOCIOCULTURAL}

Es un conjunto de técnicas sociales que, basándose en una pedagogía participativa, promueve actividades voluntarias - de tipo sociocultural - en el seno de un grupo o comunidad, para favorecer su desarrollo y una mejora en su calidad de vida ${ }^{19}$. Es una actividad relacionada con una serie de prácticas destinadas a generar estilos de participación sociocultural en el mayor número posible de personas. Surge como un procedimiento adecuado para sensibilizar e impulsar hacia las tareas de educación, y defender la identidad personal y colectiva. Con ella se pretende que los integrantes de la comunidad realicen actividades que favorezcan su proceso de educación continua. Lo importante, más que la actividad en sí, es la intencionalidad y la actitud de los participantes.

\footnotetext{
${ }^{18}$ El éxito del animador dependerá de la capacidad de establecer y guardar una distancia óptima entre él y la comunidad: involucrarse sin perder la perspectiva.

${ }^{19}$ Cfr. ANDER-EGG, E. Metodología y Práctica de la Animación Socio-cultural, p.91.
} 


\subsection{CALIDAD DE VIDA}

Cada comunidad presenta un grado de desarrollo ${ }^{20}$ que no es estático. Dentro de ella se originan múltiples cambios que han de identificarse y estudiarse — con instrumentos metodológicos oportunos - para dirigirlos y organizarlos según un plan cuya finalidad no sea el mero bienestar social (programas, prestaciones y servicios para proveer los satisfactores básicos), sino el logro de una mejor calidad de vida.

El término calidad de vida se pone de moda en los años sesenta, como respuesta al deterioro económico y ambiental, efecto no deseado de la industrialización. Se refiere no sólo a un estándar económico, sino a la existencia de condiciones y posibilidades reales para el desarrollo de toda persona, de toda la persona y de todas las personas ${ }^{21}$. En este punto es necesario evitar posturas demagógicas que propongan un utópico modelo de desarrollo lineal, o tener como meta alcanzar determinados índices económicos de bienestar que ya otros han logrado...

Desde tal óptica el desarrollo comunitario tendría muy poco que hacer, ya que (...) se trataría de una perspectiva fundamentalmente exógena, asistencial, de mera financiación de un retraso (denominado subdesarrollo) con la mirada puesta en unas metas u objetivos (elevar la renta per capita) que tienen con frecuencia poco que ver con la situación social, política y económica en que vive una determinada comunidad y de la cual se deriva la mayoría de las veces esa situación de retraso ${ }^{22}$.

Calidad de vida es un modelo alternativo de sociedad en el que se da prioridad a la satisfacción de las auténticas necesidades humanas, antes que a las exigencias de una economía floreciente.

\footnotetext{
${ }^{20}$ El desarrollo se relaciona con el nivel de ingreso, los sistemas de producción, la organización y movilidad social, los servicios públicos, los modos de convivencia y de comunicación, etcétera.

${ }^{21}$ ANDER-EGG, E. Introducción al Trabajo Social, p.92.

22 REQUEJO OSORIO, Agustín. Desarrollo Comunitario y Educación, en Iniciativas Sociales en Educación Informal, p.351.
} 
Tal modelo configura un tipo de ciudadano capaz de pensar globalmente y actuar localmente ${ }^{23}$, gracias a su desarrollo espiritual - expresado en un alto grado de cultura, efectividad en la organización social y amplitud de horizontes-, todo esto vinculado al desarrollo material que implica un nivel de vida con soporte técnico, notoria civilización y recursos económicos suficientes ${ }^{24}$.

Antes de referirnos a las categorías que expresan la calidad de vida, presentamos un esquema que sintetiza los contenidos fundamentales de lo expuesto hasta aquí:

\section{ESQUEMA 1}

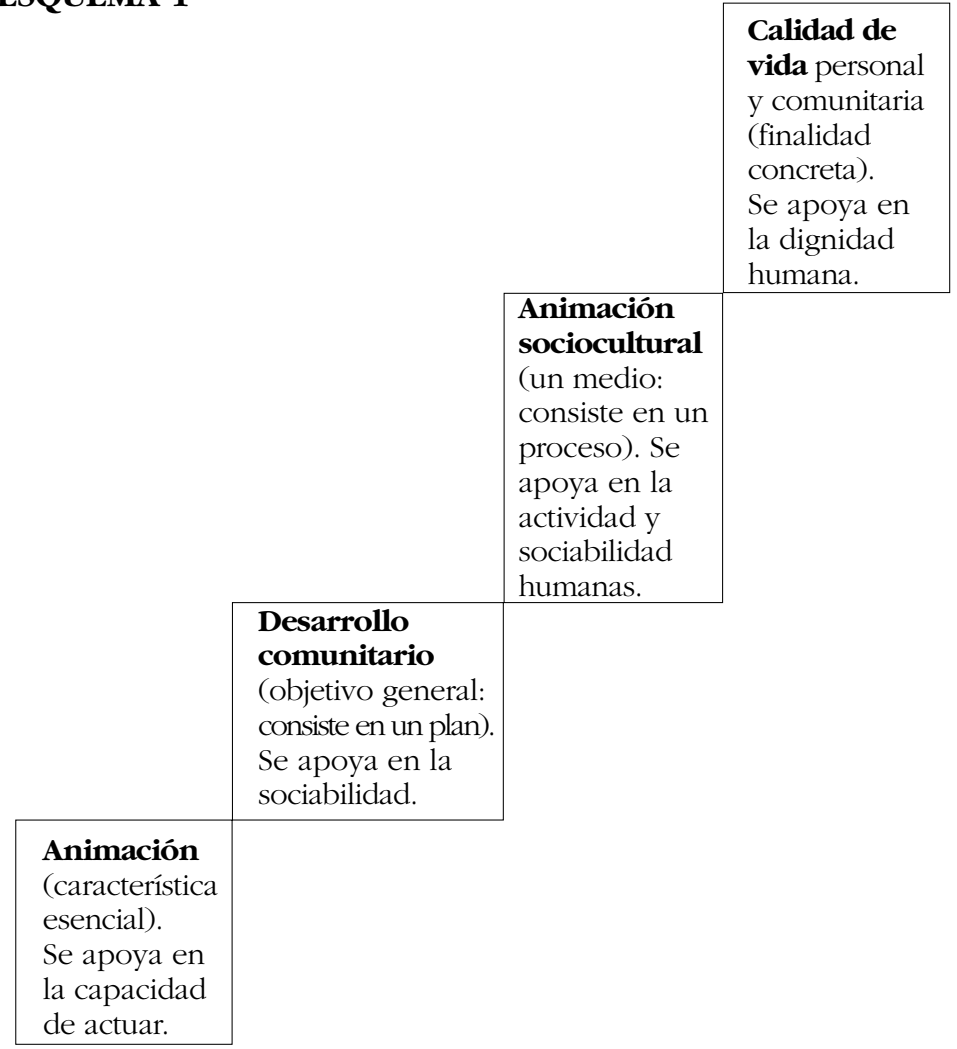

${ }^{23}$ Se trata de dar más importancia a lo local y de insistir en que las propuestas de las políticas sociales atiendan a las necesidades y problemas de los sectores populares.

${ }^{24}$ Cfr. ANDER-EGG, E. Introducción al Trabajo Social, p.91 a 95. 


\section{PRINCIPALES CATEGORÍAS ${ }^{25}$ DE LA CAIIDAD DE VIDA}

Es un hecho que, pese el razonable deseo de utilizar una metodología cada vez más objetiva para medir y comparar el desarrollo humano, las categorías de excelencia no siempre son equiparables. No existe un camino trazado por el cual los distintos grupos humanos hayan de circular —antes o después, más rápido o más lento- para desarrollarse. Por eso, las comparaciones más justas y reveladoras han de hacerse por defecto: a mayor disfunción social, menor salud ${ }^{26}$.

Además, en el trasfondo de todos los indicadores están latentes las necesidades de ser y de poseer, así como los satisfactores que las cubren ${ }^{27}$. De esta manera, la permanencia y subsistencia —necesidades de ser- se satisfacen con la alimentación, vestido y vivienda; las de poseer, concretamente, protección, afecto, entendimiento, participación y ocio, se cubren, entre otros satisfactores, con la salud —individual y social-, la educación y la investigación.

Adoptaremos algunas categorías ${ }^{28}$ para obtener indicios de la salud social, entendida como la gradación de los estados de felicidad colectiva o excelencia grupal en ámbitos sociales -familia, asociaciones, comunidad, etcétera- donde más fácilmente pueden optimizarse y minimizarse los conflictos ${ }^{29}$. Ciertas medidas investigan el defecto ${ }^{30}$, para calibrar la cantidad y calidad de desviaciones percibidas como tales por el entorno social ${ }^{31}$.

\footnotetext{
${ }^{25}$ Las categorías se refieren tanto a realizaciones materiales como a cambios de actitud.

${ }^{26}$ Cfr. PÉREZ ADÁN, José. La Salud Social, p.32.

${ }^{27}$ Cfr. COLOMER, Antonio (Coord.) Sociedad Solidaria y Desarrollo Alternativo, en LÓPEZ DE LLERGO, Ana Teresa, Valores, Valoraciones y Virtudes, p.139.

${ }^{28}$ Cfr. PÉREZ ADÁN, J. Op. cit, p.17 y 118 y ss.

${ }^{29}$ Cfr. Idem, p.14 y 15 .

${ }^{30}$ Deben revisarse las conductas disfuncionales y desincentivarse, incluso con medidas coercitivas.

${ }^{31}$ En Sociología, el estudio de la desviación ha de considerar conceptos como el de civilidad o derechos sociales.
} 
$1^{\circ}$. Equidad generacional: mide los índices de solidaridad entre generaciones a través del examen de baremos de calidad de vida y soporte afectivo próximo en la primera y tercera edad. Señala la disfuncionalidad según el número de personas mayores de 65 años que viven solas, y la equidad, de acuerdo con la dedicación personalizada en número de horas, y de convivencia que recibe cada menor de 15 años de sus padres y abuelos.

$2^{\circ}$. Desigualdad: mide por un lado, los grados de bienestar estandarizable y, por otro, los grados de seguridad proyectados también en el futuro, a través de la esperanza de vida. Se refiere a la esperanza de vida al nacer y a la esperanza de vida total, así como al costo social familiar para la alimentación, vivienda, infraestructura social, educación y sanidad.

$3^{\circ}$. Deuda filial diacrónica: mide las acciones sociales de efectos diferidos catalogados como perjudiciales (la cuantificación de la deuda ambiental). Aquí se suman: la conciencia ecológica que se obtiene como dato disfuncional (negativo) con la media de consumo de energía no renovable, per cápita anual; más la media de desecho de acumulación dañina, per cápita anual. Esto se suma al ahorro e inversión social familiar, que propicia la creación de patrimonio, de dote y de seguros transmisibles donde los haya. El indicador nos da idea de la razón social y del espíritu de comunidad que prevalece.

$4^{\circ}$. Conciencia cívica: aquí se miden tres pautas sociales, la corresponsabilidad fiscal y la prestación social, por un lado; por otro, el respeto mutuo medible por defecto en el índice de criminalidad (delitos legalmente considerados), y por ultimo, la participación formal e informal en todos los ámbitos sociales. Hablamos de participación económica que se expresa en el dato disfuncional de la concentración de propiedad, y de participación sociopolítica según el número de horas no remuneradas dedicadas a actividades solidarias.

$5^{\circ}$. Pluralidad: se mide por la presencia de asociaciones representativas, y por la capacidad de libre adscripción y circulación entre comunidades y sociedades intermedias, así 
como la permeabilidad de las barreras sociopolíticas. Se suma la movilidad social (variación de estatus de tres generaciones en la misma familia) y la diversidad. Esta última comprende tres variables: diversidad genérica (tareas domésticas realizadas por varones), diversidad espacial (porcentaje de población rural) y diversidad de origen (población foránea).

Si relacionamos las categorías propuestas por José Pérez Adán con los conceptos definidos en esta investigación, tendremos el esquema siguiente, elaborado por nosotros para fines didácticos:

\section{ESQUEMA 2}

\begin{tabular}{|c|c|c|}
\hline CATEGORIAAS & $\begin{array}{l}\text { ASPECTOS QUE } \\
\text { LAS CONFORMAN }\end{array}$ & $\begin{array}{l}\text { BENEFICIOS QUE } \\
\text { PUEDEN CONSEGUIRSE }\end{array}$ \\
\hline $\begin{array}{l}\text { Equidad } \\
\text { generacional }\end{array}$ & $\begin{array}{l}\text { Solidaridad } \\
\text { intergeneracional: } \\
\text { cuidado de los } \\
\text { ancianos y los niños. }\end{array}$ & $\begin{array}{l}\text { Promoción de } \\
\text { la calidad de vida } \\
\text { por medio de } \\
\text { la familia soberana. }\end{array}$ \\
\hline Desigualdad & $\begin{array}{l}\text { Bienestar presente. } \\
\text { Esperanza de } \\
\text { buen futuro. }\end{array}$ & $\begin{array}{l}\text { Promoción del } \\
\text { desarrollo comunitario } \\
\text { al crear condiciones } \\
\text { que beneficien a } \\
\text { todos, incluyendo a } \\
\text { los no nacidos. }\end{array}$ \\
\hline $\begin{array}{l}\text { Deuda filial } \\
\text { diacrónica }\end{array}$ & $\begin{array}{l}\text { Cuidado del } \\
\text { ambiente físico } \\
\text { y espiritual. }\end{array}$ & $\begin{array}{l}\text { Promoción -a través } \\
\text { de la animación } \\
\text { sociocultural- de un } \\
\text { entorno adecuado y rico } \\
\text { en cultura y tradiciones. }\end{array}$ \\
\hline Conciencia cívica & $\begin{array}{l}\text { Corresponsabilidad } \\
\text { fiscal y prestaciones } \\
\text { sociales. } \\
\text { Respeto mutuo. } \\
\text { Participación formal } \\
\text { e informal en todos } \\
\text { los ámbitos. }\end{array}$ & $\begin{array}{l}\text { Cristalización de la } \\
\text { calidad de vida } \\
\text { plena en el yo } \\
\text { individual. }\end{array}$ \\
\hline
\end{tabular}




\begin{tabular}{|l|l|l|}
\hline Pluralidad & Asociaciones & $\begin{array}{l}\text { Cristalización de la } \\
\text { calidad de vida en }\end{array}$ \\
& representativas. & el yo colectivo. \\
Libre adscripción & \\
y circulación en & \\
las asociaciones. & \\
Permeabilidad para & \\
& acceder a otros estatus \\
& socioeconómicos. & \\
& & \\
\hline
\end{tabular}

Ya hemos analizado los aspectos que conforman las categorías; ahora explicaremos los síntomas de salud social que se expresan en beneficios individuales y colectivos, cuando el balance de las categorías es favorable.

En relación con la equidad generacional, los cimientos de la calidad de vida se dan gracias a la familia soberana, porque ahí es real la diacronía entre ancianos y niños; se ofrece seguridad actual a los miembros del grupo, y cobertura permanente de las demandas afectivas. Todo ello favorece la civilidad en los distintos ámbitos sociales.

En cuanto a la desigualdad ${ }^{32}$, la salud social se expresa en el bienestar del colectivo humano porque para todos hay alimentos, vivienda, infraestructura adecuada, educación y servicios sanitarios. Además, tales satisfactores se proyectan en el tiempo, pues existen los suficientes recursos — materiales y humanos - para generarlos. Esto hace viable el desarrollo comunitario.

La deuda filial diacrónica consiste en el compromiso del cuidado del ambiente físico y espiritual para ofrecer un lugar adecuado y rico en cultura y tradiciones, donde la animación

\footnotetext{
${ }^{32}$ El factor condicionante más claro de desigualdad es la esperanza de vida, pero ha de contarse desde el inicio de la vida y no sólo desde el nacimiento. El peligro que amenaza la sustracción del tiempo prenatal del control social es la aparición de una práctica médica fascista. Cfr. PÉREZ Adán, J. Op. cit, p.29 y 30 .
} 
sociocultural verdaderamente promueva el desarrollo. Los beneficios tangibles son la capacidad de ahorro e inversión económica, y un entorno ecológico adecuado; los intangibles son múltiples, como la preocupación por descubrir formas para cuidar el ambiente, evitar la corrupción, pornografía, etcétera.

La conciencia cívica cristaliza el más alto nivel de desarrollo comunitario reflejado en la calidad de vida de ciudadanos virtuosos con deseos de incorporar voluntariamente, a su quehacer cotidiano, actividades solidarias y desinteresadas. Esto manifiesta la riqueza y plenitud del yo individual.

La pluralidad requiere de variadas asociaciones representativas intermedias, y de flexibilidad para adscribirse a unas u otras, de acuerdo con las cambiantes circunstancias de las personas.

Tales asociaciones han de $^{33}$ :

- Postular una cultura y una ética de solidaridad.

- Actuar en un plano democrático, tanto en la formulación de sus objetivos como en la distribución de las responsabilidades directivas.

- Hacer vivir una actitud de gratuidad a través del voluntariado y de las finalidades no lucrativas.

- Promover la capacidad de proyectarse para conseguir un fin asumido como válido y mejorar a la comunidad, mediante la acción directa, el control y la evaluación.

- Contrarrestar la politización, corrupción y apatía.

- Favorecer la obtención de recursos.

- Propiciar la participación de todos.

Para todo ello se requieren tres niveles de ciudadanos activos: voluntarios, técnicos y expertos. Los principales criterios de selección de voluntarios son la experiencia y competencia profesionales, las cualidades humanas, el respeto a los demás y la capacidad de convivir con otros. Así, la comunidad será enriquecedora por las oportunidades que ofrece, y será tangible y real la posibilidad de acceder a mejores formas de calidad de vida. Esto dará plenitud al yo colectivo.

${ }^{33}$ Cfr. QUinTANA CABANAS, José María. Op. cit, p.82. 
En el esquema 3 presentamos algunas propuestas para que el desarrollo comunitario incida en la calidad de vida individual y grupal.

\section{ESQUEMA 3}

\begin{tabular}{|c|c|}
\hline CATEGORÍAS & PROPUESTAS \\
\hline $\begin{array}{l}\text { Equidad } \\
\text { generacional }\end{array}$ & $\begin{array}{l}\text { - Fortalecer -en el seno familiar- } \\
\text { la identidad personal y social para } \\
\text { forjar ciudadanos seguros y solidarios. }\end{array}$ \\
\hline Desigualdad & $\begin{array}{l}\text { - Promover una sociedad más humana } \\
\text { y civilizada, mediante la formación } \\
\text { de comunidades de ciudadanos } \\
\text { responsables, preocupados por el } \\
\text { bienestar social y atentos a resolver } \\
\text { problemas colectivos - presentes y } \\
\text { futuros- de un modo solidario. Han } \\
\text { de ser defensores de la vida humana } \\
\text { (vs. aborto y eutanasia). }\end{array}$ \\
\hline $\begin{array}{l}\text { Deuda filial } \\
\text { diacrónica }\end{array}$ & $\begin{array}{l}\text { - Superar las acciones ecológicas es- } \\
\text { porádicas e inconexas, y promover } \\
\text { aquellas que benefician a todos en el } \\
\text { presente y el futuro }{ }^{34} \text {. } \\
\text { - Potenciar el cooperativismo, las } \\
\text { campañas informativas y las actividades } \\
\text { culturales. Favorecer la formación y } \\
\text { cohesión de grupos, la raigambre de } \\
\text { las personas con su medio, la afirma- } \\
\text { ción de la identidad cultural, la autosu- } \\
\text { ficiencia (por ejemplo, capacidad de } \\
\text { ahorro) en los principales ámbitos } \\
\text { de la vida, la motivación y el estímulo. }\end{array}$ \\
\hline
\end{tabular}

${ }^{34}$ En un plan ecológico hay que promover el uso racional de los recursos de la comunidad y la cooperación de todos. Tal cooperación debe ser estimulada y coordinada por el gobierno, pero no hay que esperar todo del Estado. Lo conveniente es preparar a la comunidad para organizarse y para aceptar las nuevas soluciones. 


\begin{tabular}{|c|c|}
\hline $\begin{array}{l}\text { Conciencia } \\
\text { cívica }\end{array}$ & $\begin{array}{l}\text { - Promover, mediante la participación } \\
\text { ciudadana, la existencia de una politica } \\
\text { social-incentivos fiscales, prestaciones, } \\
\text { etcétera-para mejorar la calidad de vida. } \\
\text { - Fomentar la actividad local sin } \\
\text { descuidar lo global. } \\
\text { - Evitar todas las formas de autorita- } \\
\text { rismo, paternalismo y manipulación. } \\
\text { - Transformar, desde abajo, la sociedad } \\
\text { política y desarrollar nuevos estilos de } \\
\text { vida, mediante la mayor participación } \\
\text { de la sociedad civil. } \\
\text { - Desincentivar coercitivamente con- } \\
\text { ductas antisociales (criminalidad, } \\
\text { etcétera). } \\
\text { - Impulsar la autosuficiencia a partir } \\
\text { de los recursos propios -personales } \\
\text { y comunitarios- bien explotados e } \\
\text { inteligentemente aprovechados. } \\
\text { (desarrollo endógeno vs. exógeno). }\end{array}$ \\
\hline Pluralidad & $\begin{array}{l}\text { - Considerar factores estructurales y } \\
\text { socioculturales. } \\
\text { - Aprovechar símbolos, tradiciones, } \\
\text { folkore, efemérides, etcétera. } \\
\text { - Evitar la emulación indiscriminada } \\
\text { de patrones extranjeros y de índices } \\
\text { económicos que otros han logrado. } \\
\text { - Fomentar asociaciones -con estruc- } \\
\text { tura, continuidad, fines conscientes y } \\
\text { concretos- que encaucen la tendencia } \\
\text { natural de las personas a reunirse. Si } \\
\text { es posible, obtener un reconocimiento } \\
\text { jurídico. }\end{array}$ \\
\hline
\end{tabular}




\begin{tabular}{|l|l|}
\hline & • Identificar y formar a los líderes, y \\
conocer su radio de influencia. \\
• Promover la participación ciudadana \\
en las formas de gobierno local, así \\
los cambios no serán bruscos sino \\
progresivos y reglamentados. \\
- Favorecer la participación de \\
mujeres y jóvenes. (amplía la base, \\
garantiza el progreso).
\end{tabular}




\section{BIBLIOGRAFÍA}

ANDER-EGG, Ezequiel.

- Introducción al Trabajo Social, Editorial El Ateneo, México, 1993.

- Metodología y Práctica del Desarrollo Comunitario. ¿Qué es el Desarrollo de la Comunidad?, Vol. I, Editorial LumenHumanitas, Argentina, 1998.

- Reflexiones en torno a los Métodos del Trabajo Social, Editorial El Ateneo S.A. de C.V., México, 1992.

ESCALANTE F. Rosendo. MIÑANO, Max. Investigación, Organización y Desarrollo de la Comunidad, Ediciones Oasis, S.A., México, 1990.

KISNERMAN, Natalio. La Comunidad, Editorial Humanitas, Buenos Aires, 1984.

LÓPEZ DE LLERGO, Ana Teresa. Valores, Valoraciones y Virtudes, Editorial CECSA, Patria Cultural, México, 1999.

PÉREZ ADÁN, José. La Salud Social, Editorial Trotta, Madrid, 1999.

QUINTANA CABANAS, José María. Los Ámbitos Profesionales de la Animación, Narcea S.A. de Ediciones, Madrid, 1993. REQUEJO OSORIO, Agustín. Desarrollo Comunitario y Educación, en Iniciativas Sociales en Educación Informal, Ediciones RIALP, S.A. Madrid, 1991. 